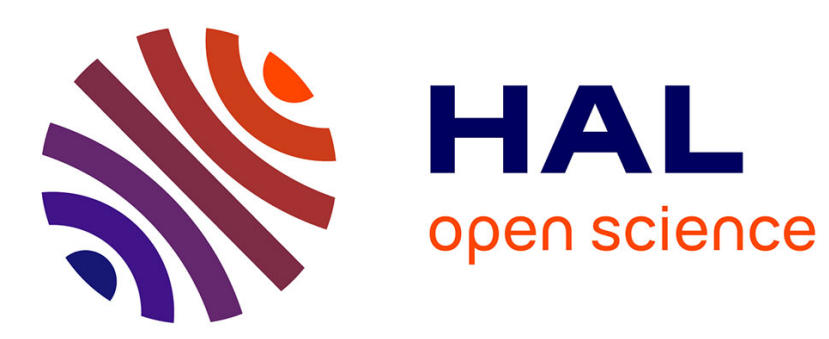

\title{
A self-avoiding walk on random strips
}

Bernard Derrida

\section{To cite this version:}

Bernard Derrida. A self-avoiding walk on random strips. Journal of Physics A : Mathematical and

General, 1982, 15 (3), pp.L119-L125. 10.1088/0305-4470/15/3/008 . hal-03285935

\section{HAL Id: hal-03285935 \\ https://hal.science/hal-03285935}

Submitted on 19 Jul 2021

HAL is a multi-disciplinary open access archive for the deposit and dissemination of scientific research documents, whether they are published or not. The documents may come from teaching and research institutions in France or abroad, or from public or private research centers.
L'archive ouverte pluridisciplinaire HAL, est destinée au dépôt et à la diffusion de documents scientifiques de niveau recherche, publiés ou non, émanant des établissements d'enseignement et de recherche français ou étrangers, des laboratoires publics ou privés. 


\title{
LETTER TO THE EDTOR
}

\section{A self-avoiding walk on random strips}

\author{
B Derrida \\ SPT, CEN-Saclay, 91191 Gif-sur-Yvette Cedex, France
}

Received 27 November 1981

\begin{abstract}
To describe a polymer in a random medium, we consider a self-avoiding walk (SAW) on a lattice whose bonds are randomly favourable (with probability $1-p$ ) or unfavourable (with probability $p$ ). The size of a sAW of $N$ steps is calculated when the lattice is a strip by performing products of random matrices. When the weight of unfavourable bonds tends to 0 , there exist critical concentrations $p_{c}$ where the size of the SAW undergoes first-order transitions. The origin of these transitions is explained as well as the fact that the limits $p \rightarrow 0$ or $p \rightarrow 1$ are discontinuous. Possible consequences for higher-dimensional systems are discussed.
\end{abstract}

According to Harris (1974), the critical properties of a physical system are modified by the presence of impurities if the exponent $\alpha$ is positive. It is the case of polymers below dimension 4 ( $\alpha \simeq 0.236$ in $d=3$ and $\alpha \approx 0.5$ in dimension 2) (Chakrabarti and Kertész 1981 and references therein). So one expects that the properties of a long polymer are modified by the presence of quenched impurities (which can be either attractive or repulsive for the chain). In particular, the exponent $\nu$ which relates the size $R$ of a polymer to its molecular weight $N\left(R^{2} \sim N^{2 v}\right)$ should be changed by the presence of these impurities.

However by studying a simple model of a polymer in a random medium, one could believe that the randomness has no effect. This model is the $n \rightarrow 0$ limit of the Heisenberg model on a lattice with random nearest-neighbour ferromagnetic interactions $J_{i j}$

$$
\mathscr{H}=-\sum_{(i j)} J_{i} \boldsymbol{S}_{i} \cdot \boldsymbol{S}_{i} \cdot
$$

The $J_{i j}$ are random positive numbers distributed according to a probability distribution $\rho\left(J_{i j}\right)$. Exactly as in the pure case (Daoud et al 1975), the correlation function $\left\langle S_{0} \cdot S_{R}\right\rangle$ gives in the limit $n \rightarrow 0$ the generating function $G_{0 R}$ of the self-avoiding walks (SAw) whose ends are 0 and $\boldsymbol{R}$.

$$
\lim _{n \rightarrow 0}\left\langle S_{0} \cdot S_{R}\right\rangle=G_{0 R}\left(\left\{J_{i j}\right\}\right)=\sum_{\left\{0, i_{1}, i_{2}, \ldots, i_{N-1}, R\right\}} J_{0 i_{1}} J_{i_{1} i_{2}} \ldots J_{i_{N-1} R} .
$$

Here $\left\{0, i_{1}, i_{2}, \ldots, i_{N-1}, \boldsymbol{R}\right\}$ represents a sAw of $N$ steps which passes through sites $i_{1}, i_{2}, \ldots, i_{N-1}$. The sum (2) runs over all the sAw going from 0 to $\boldsymbol{R}$. Because each interaction $J_{i j}$ appears in (2) only with power 0 or 1 , the average of $G_{0 R}$ is given by

$$
\overline{G_{0 R}}=\sum_{N} \mathcal{N}_{0 R}(N)(\bar{J})^{N}
$$


where $\mathcal{N}_{\mathrm{OR}}(N)$ is the number of SAW of $N$ steps going from 0 to $\boldsymbol{R}$. So the average correlation function $\overline{G_{0 R}}$ is identical to the correlation function of the pure system where all the $J_{i j}$ are replaced by their average $\bar{J}$. This identity (3) does not contradict the Harris criterion because, for large $R$, the quantity which is meaningful to average is the logarithm of $G_{0 R}$ (Derrida and Hilhorst 1981). To average this logarithm is a difficult task and up to now it has never been done for polymers. For the numerical results presented in this letter, the calculations were done by performing products of random transfer matrices and the average was done automatically by taking long enough strips.

The distribution $\rho\left(J_{i j}\right)$ chosen in the present work is a sum of two delta functions:

$$
\rho\left(J_{i j}\right)=(1-p) \delta\left(J_{i j}-x\right)+p \delta\left(J_{i j}-x a\right) .
$$

This represents a lattice formed of two kinds of bonds: normal bonds with a concentration $1-p$ and unfavourable bonds (if $a<1$ ) with concentration $p$. The parameter $a$ is a weight given to each unfavourable bond. A given configuration of a SAW of $N$ steps which passes through $N_{1}$ normal bonds and $N_{2}=N-N_{1}$ unfavourable bonds has a weight $a^{N_{2}}$.

The average size $\left\langle R^{2}\right\rangle$ of a SAW of $N$ monomers with a fixed origin 0 is given by

$$
\left\langle R^{2}\right\rangle=\left(\sum_{R, N_{2}} a^{N_{2}} \boldsymbol{R}^{2} \mathcal{N}_{0 R}\left(N, N_{2}\right)\right)\left(\sum_{R, N_{2}} a^{N_{2}} \mathcal{N}_{0 R}\left(N, N_{2}\right)\right)^{-1}
$$

where $\mathcal{N}_{0 R}\left(N, N_{2}\right)$ is the number of SAW of $N$ steps going from 0 to $R$ and passing through $N_{2}$ unfavourable bonds. Another quantity of interest is the number of unfavourable bonds $\left\langle N_{2}\right\rangle$ on the walk

$$
\left\langle N_{2}\right\rangle=\left(\sum_{\boldsymbol{R}, N_{2}} a^{N_{2}} N_{2} \mathcal{N}_{0 \boldsymbol{R}}\left(N, N_{2}\right)\right)\left(\sum_{\boldsymbol{R}, N_{2}} a^{N_{2}} \mathcal{N}_{0 \boldsymbol{R}}\left(N, N_{2}\right)\right)^{-1} .
$$

Because the lattice is formed of random bonds, the $\mathcal{N}_{0 R}\left(N, N_{2}\right)$ depend on the local arrangement of bonds around the site 0 . However, in the limit of long chains $(N \rightarrow \infty)$, the polymer can explore a very large part of the lattice and it is reasonable to assume that the effect of this local arrangement is washed out. Therefore, one expects that in this limit $N \rightarrow \infty,\left\langle R^{2}\right\rangle$ and $\left\langle N_{2}\right\rangle$ depend only on $N, a$ and $p$ in the following way

$$
\langle R\rangle=\left\langle R^{2}\right\rangle^{1 / 2} \sim N^{\nu(p, a)} / A(p, a) \quad\left\langle N_{2}\right\rangle \sim N B(p, a)
$$

where $\nu, A$ and $B$ are functions of $a$ and $p$ only. Of course, the first physical quantity one should like to calculate is $\nu(p, a)$ for lattices in two and three dimensions. Unfortunately, one does not know how to do it directly. Thus it is interesting to study the problem on strips with the intention of using finite size scaling to obtain the behaviour of two- or three-dimensional systems.

The simplest way to study one-dimensional systems is to use transfer matrices. For polymers, the idea of a transfer matrix was first introduced by Klein (1980) and later in a work on the phenomenological renormalisation (Derrida 1981). From the eigenvalues of the transfer matrix, one can calculate the physical quantities of interest. The generalisation of the transfer matrix to random lattices is straightforward. However, one has to work with products of random matrices and there does not exist a simple way to calculate the Liapounov numbers which play the role of the eigenvalues 
for products of random matrices. In most cases, the only way to calculate the properties of random one-dimensional systems is to generate the transfer matrices at random and to do the product numerically. To eliminate the fluctuations due to a particular sample of matrices, the product has to be as long as possible.

For more details about the study of disordered systems by products of random matrices, I refer to the work of Pichard and Sarma (1981) who have used this method for the problem of localisation.

The quantity which can be obtained directly from this product of random matrices is the generating function $G_{O R}$. If the points 0 and $\boldsymbol{R}$ are far from each other, one finds that this generating function behaves like

$$
G_{0 R} \sim \exp (R \mu(x, p, a)) .
$$

Then by applying to the random case the ideas worked out by Klein (1980) for pure lattices, one can calculate the average size $\langle R\rangle$ and the number of unfavourable bonds $\left\langle N_{2}\right\rangle$ of a walk of $N$ monomers

$$
\begin{aligned}
& N=\left.x_{\mathrm{c}} \frac{\partial \mu}{\partial x}\right|_{x_{\mathrm{c}}}\langle R\rangle=A(p, a)\langle R\rangle \\
& \left\langle N_{2}\right\rangle=\left.\langle R\rangle a \frac{\partial \mu}{\partial a}\right|_{x_{\mathrm{c}}}=N B(p, a)
\end{aligned}
$$

where $x_{\mathrm{c}}$ is the value of $x$ where $\mu(x, p, a)$ vanishes

$$
\mu\left(x_{\mathrm{c}}, p, a\right)=0 .
$$

The size $\langle R\rangle$ is proportional to $N$ as it should be for a sAw on a one-dimensional lattice. In figure $1(a)$, the circles and the stars represent the function $A(p, a)$ as a function of $p$ for a strip of width 2 with periodic boundary conditions. The weight $a$ of the unfavourable bonds was either $10^{-12}$ (circles) or $10^{-48}$ (stars). These two curves show a rather complicated behaviour as a function of $p$. First, the two curves do not coincide everywhere and this means that $a=10^{-12}$ or $10^{-48}$ is not small enough to reach the asymptotic limit $a \rightarrow 0$. Another surprise is that in the limit $p \rightarrow 1$, one does not recover the value of the pure system. One can show (Derrida 1982) that $A(p, a)$ is in fact continuous at $p=1$, but the slope is proportional to $\log a$. One has also to understand the origin of the maximum around $p=0.2$ and of the irregularity around $p=0.75$. The maximum is due to a competition between the fact that the saw tries to avoid the unfavourable bonds and the entropy which tends to spread the sAw. We shall see that in the limit $a \rightarrow 0$, this maximum disappears. The most interesting result is the irregularity around $p=0.75$. In the limit $a \rightarrow 0$, it becomes a phase transition that we shall discuss further.

One should notice that these results show clearly that the most probable value of $G_{0 R}$ is not equal to its average $\overline{G_{0 R}}$ (Derrida and Hilhorst 1981). If $G_{0 R}$ could be replaced by its average in equation $(8), A(p, a)$ would be an horizontal line $\left(A(p, a)=\frac{3}{2}\right)$. Here the calculations have been done for strips of length 10000 without averaging. The smoothness of the curves is an indication that the statistical errors are small.

In figure $2(a)$, the circles and the stars represent the function $A(p, a)$ as a function of $p$ for a strip of width 3 with periodic boundary conditions $\left(a=10^{-12}\right.$ corresponds to the circles and $a=10^{-48}$ to the stars). The results are very similar to those for a strip of width 2 . One difference is that the irregularity around $p=0.7$ seems to be 


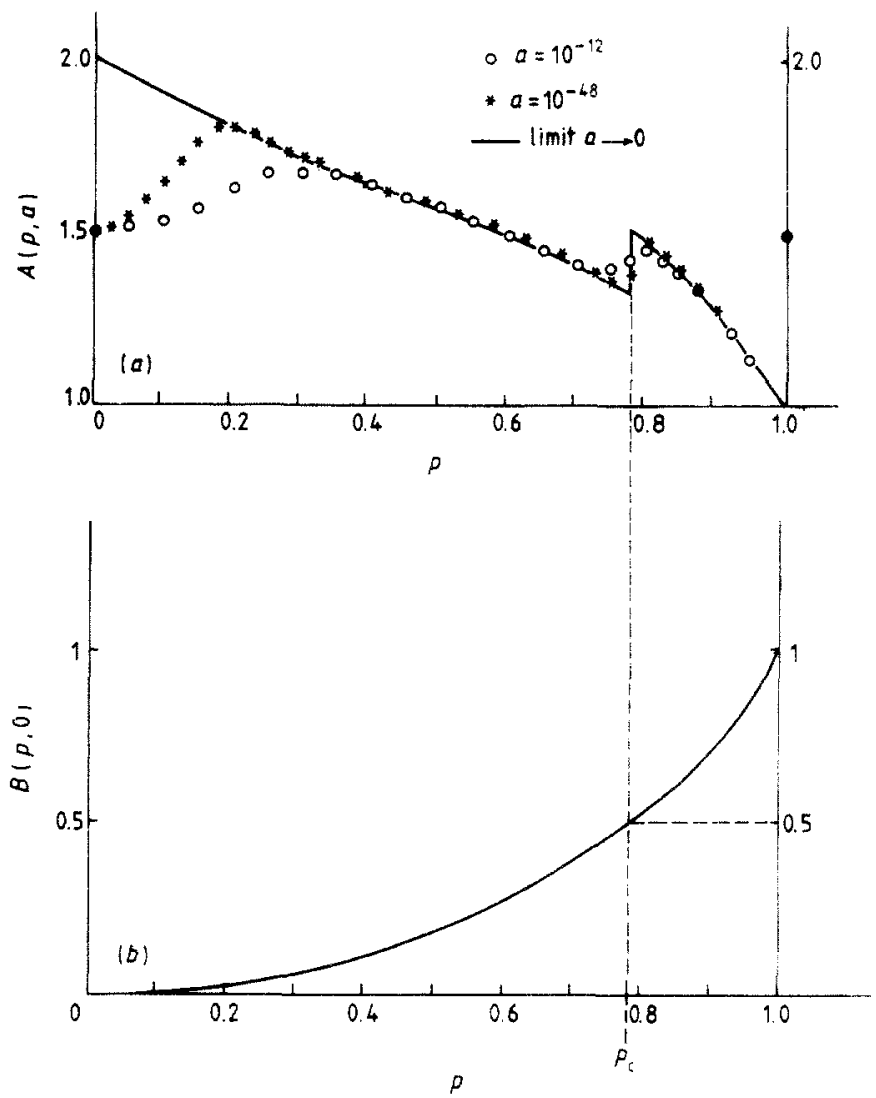

Figure 1. The circles and the stars represent the function $A(p, a)$ for two values of $a=10^{-12}$ and $a=10^{-48}$ in the case of a strip of width 2 with periodic boundary conditions. The curves represent $(a) A(p, a)$ and $(b) B(p, a)$ in the limit $a \rightarrow 0$. We see the transition at $p_{\mathrm{c}}=\frac{1}{4}(\sqrt{17}-1)$.

more complicated. We shall see that for a strip of width 3 , the function $A(p, a)$ has several jumps in the limit $a \rightarrow 0$. Another difference is that the maximum $p=0.35$ is more pronounced than for a strip of width 2 . Preliminary results for strips of larger width (4 and 5) show that this maximum becomes sharper and sharper with increasing width.

The problem becomes simpler in the limit $a \rightarrow 0$. If there is percolation of favourable bonds, it is the problem of a SAw on a diluted lattice. However even if there is no percolation of favourable bonds, the problem is well defined. The only configurations allowed for a SAw of length $N$ starting at point 0 are those for which $N_{2}$ is minimum. This means that the walk tries to pass through the maximum number of favourable bonds with the constraint to remain a self-avoiding walk. This limit $a \rightarrow 0$ is simpler because the pioduct of random matrices is reduced to a Markov process: in each term of the product of random matrices it is sufficient to know the dominant power of $a$. The same kind of simplification was also used for a spin glass model in the limit of low temperatures (Derrida et al 1978).

The simplification is such that for a strip of width 2 , it is possible to obtain the analytic expression of $A(p, 0)$ and $B(p, 0)$. One finds that there exists a critical 


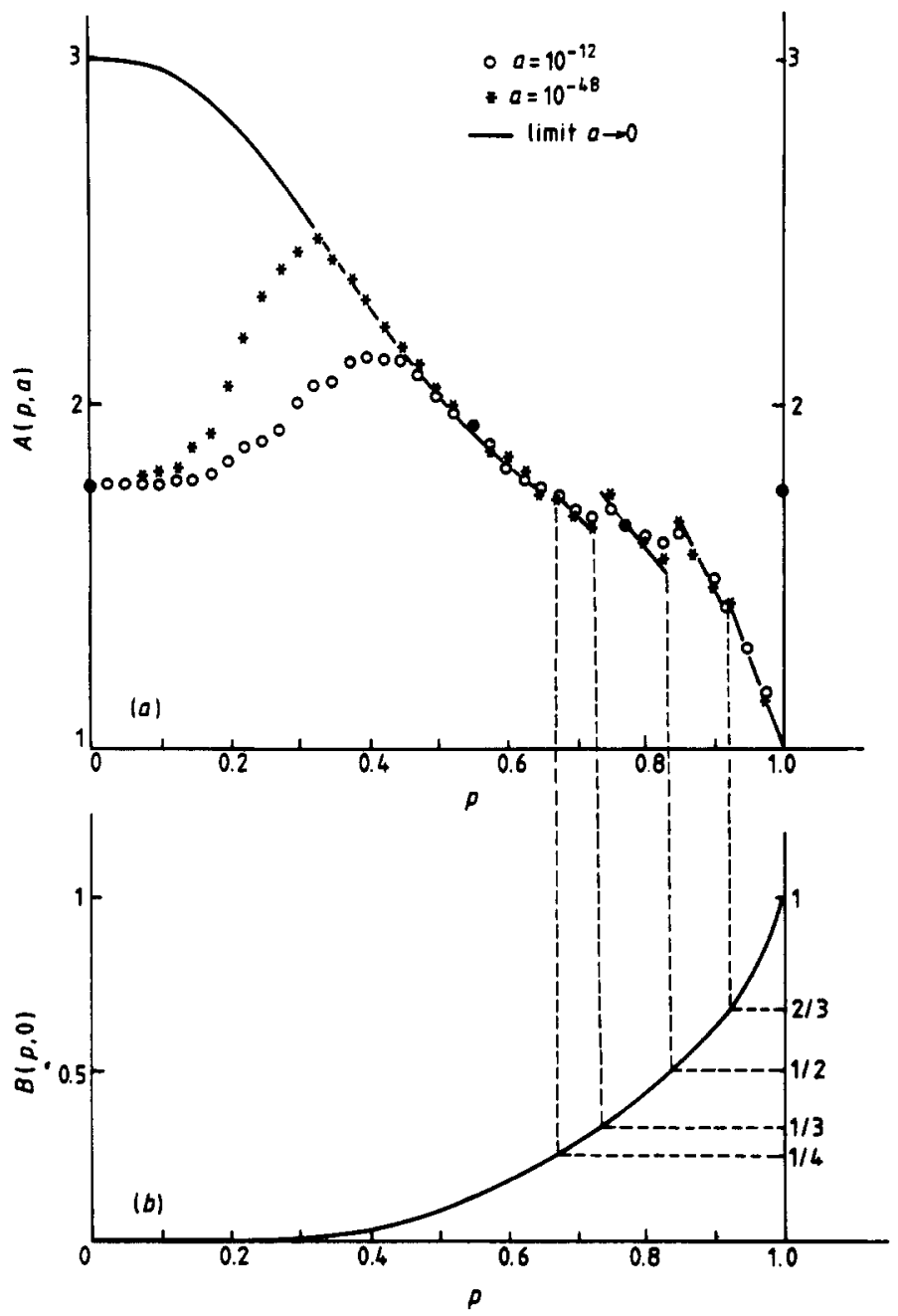

Figare 2. The same as figure 1 but for a strip of width 3 with periodic boundary conditions. We see several jumps of the function $A(p, 0)$ corresponding to simple rational values of $B(p, 0)$.

concentration $p_{c}=\frac{1}{4}(\sqrt{17}-1) \simeq 0.7808$ and that $A(p, 0)$ and $B(p, 0)$ are given by

$$
\left.\begin{array}{l}
A(p, 0)=\frac{2+p-p^{2}+6 p^{3}+p^{4}-4 p^{5}}{1+p+3 p^{3}} \\
B(p, 0)=\frac{p^{2}+p^{3}+2 p^{4}+p^{5}}{2+p-p^{2}+6 p^{3}+p^{4}-4 p^{5}}
\end{array}\right\} \quad \text { if } p<p_{\mathrm{c}}
$$

The details of this calculation will be presented elsewhere (Derrida 1982). The curves 
$A(p, 0)$ and $B(p, 0)$ are represented respectively on figures $1(a)$ and $1(b)$ (full curves). In the limit $p \rightarrow 1, A(p, 0)$ tends to unity. The reason is that when the concentration of favourable bonds is small, the SAW must be as long as possible to pass through the maximum number of favourable bonds. In the limit $p \rightarrow 0, A(p, 0)$ tends to 2 . When the concentration of unfavourable bonds is small, there exists along the strip walls of unfavourable bonds with a concentration $p^{2}$. To cross the minimum number of these walls, the SAW must be as compact as possible. The transition at $p_{\mathrm{c}}$ occurs when $B(p, 0)=\frac{1}{2}$, i.e. there are as many unfavourable bonds $\left\langle N_{2}\right\rangle$ as favourable bonds $\left\langle N_{1}\right\rangle$ on the walk. This transition can be understood by looking at figure 3. In several places along the strip, the SAW can choose between path 1 or path 2 . If $B(p, 0)<\frac{1}{2}$, the concentration of unfavourable bonds on the walk will be lowered by passing through path 1 . If $B(p, 0)>\frac{1}{2}$, this concentration is lowered by passing through path 2.

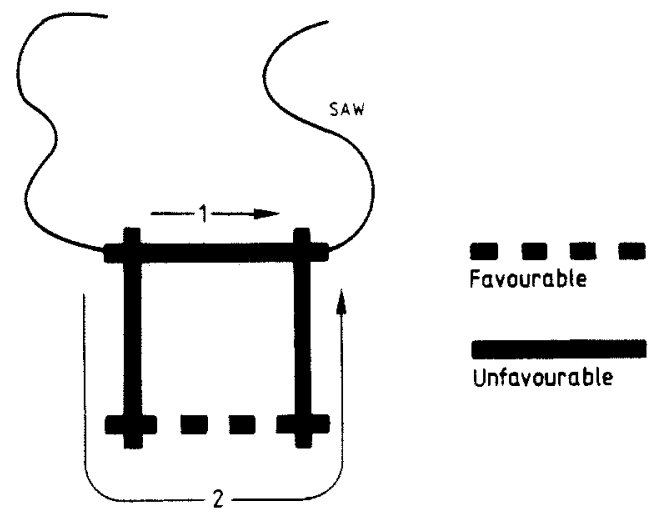

Figure 3. If the self-avoiding walk can choose between path 1 and path 2 , it will take path 1 if there are more favourable bonds on the walk than unfavourable bonds $\left(B(p, 0)<\frac{1}{2}\right)$ and path 2 otherwise. The fact that this choice occurs a macroscopic number of times along the walk is the origin of the transition.

In figures $2(a)$ and $2(b)$, the functions $A(p, 0)$ and $B(p, 0)$ are represented (full curves) for a strip of width 3 . The calculations were done by studying numerically the Markov chain for strips of length 10000 . For the same reasons as for the strip of width 2 , the SAW is as long as possible (i.e. $A(p, 0) \rightarrow 1$ ) if $p \rightarrow 1$ whereas the SAW is compact (i.e. $A(p, 0) \rightarrow 3$ ) if $p \rightarrow 0$. The main difference with the strip of width 2 is that $\boldsymbol{A}(p, 0)$ has now several jumps. This is due to the fact that for a strip of width 3 , situations similar to the one described by figure 3 occur involving configurations of more than four bonds. The jumps correspond always to values of $p$ where $B(p, 0)$ is a simple rational number.

At the end of this letter, the main questions are: to know how these results can be generalised to strips of larger widths, to bars (lattice infinite in one direction and finite in $d-1$ directions), and what are the possible consequences for infinite lattices.

For the same reasons as above, in the limit $p \rightarrow 1$ (which corresponds to a case of diluted attractive impurities) $A(p, 0) \rightarrow 1$ for strips or bars of any width. In the limit $p \rightarrow 0$ (which corresponds to a case of diluted obstacles), $A(p, 0) \rightarrow n$ for strips of width $n$ and $A(p, 0) \rightarrow n^{d-1}$ for bars of section $n^{d-1}$. Usual finite size scaling relates the $n$ dependence of $A(p, 0)$ to the exponent $\nu$ of the infinite ( $d$-dimensional) lattice 
(Daoud and de Gennes 1977, Guttmann and Whittington 1978).

$$
A(p, 0) \sim n^{1 / \nu-1} \text {. }
$$

If we assume that finite size scaling remains valid here (in particular if the limits $n \rightarrow \infty$ and $p \rightarrow 0$ commute) this would imply that $\nu=1$ for $p \rightarrow 1$ and $\nu=1 / d$ for $p \rightarrow 0$. Therefore a small amount of disorder would change $\nu$ in agreement with the Harris criterion. The prediction $\nu=1 / d$ in the limit $p \rightarrow 0$ neither agrees with a recent Monte Carlo simulation (Kremer 1981) which predicts that the exponent is not changed by the disorder, nor with a field theory approach (Chakrabarti and Kertész 1981) which argues that $\nu=\frac{1}{2}$. More work is certainly necessary to clarify this problem.

For strips of larger widths as well as for infinite lattices, the size of the SAW will have several transitions corresponding to local situations like the one shown in figure 3. These transitions will correspond to rational values of $B(p, 0)$. So they are all located in the region where the favourable bonds do not percolate (otherwise $B(p, 0)=$ $0)$.

The numerical calculations presented here were done during my visit at the Courant Institute of New York University. I should like to thank Professor J L Lebowitz for his invitation and his encouragements and the physicists of the Courant Institute for their hospitality.

\section{References}

Chakrabarti B K and Kertész J 1981 Z. Phys. B 44821

Daoud M, Cotton J P, Farnoux B, Jannink G, Sarma G, Benoit H, Duplessix R, Picot C and de Gennes P G 1975. Macromolecules 8804

Daoud M and de Gennes P G 1977 J. Physique 3885

Derrida B 1981 J. Phys. A: Math. Gen. 14 L5 1982 in preparation

Derrida B and Hilhorst H 1981 J. Phys. C: Solid State Phys. 14 L539

Derrida B, Vannimenus J and Pomeau Y 1978 J. Phys. C: Solid State Phys. 114749

Guttmann A J and Whittington S G 1978 J. Phys. A: Math. Gen. 11 L107

Harris A B 1974 J. Phys. C: Solid State Phys. 71671

Klein D J 1980 J. Stat. Phys. 23561

Kremer K 1981 Z. Phys. B 45149

Pichard J L and Sarma G 1981 J. Phys. C: Solid State Phys. 14 L127 